\title{
UNFUSED INVOLUTIONS IN FINITE GROUPS
}

\author{
Martin R. Pettet \\ Department of Mathematics \\ The University of Toledo, Toledo, Ohio 43606 \\ E-mail: mpettet@math.utoledo.edu
}

\begin{abstract}
Let $x$ be a $p$-element of a finite group $G$. We say that $x$ is unfused in $G$ if, for some Sylow $p$-subgroup $S$ of $G$ containing $x$, all $G$-conjugates of $x$ in $S$ are $S$-conjugates. It is shown (using the classification of finite simple groups) that a finite group which contains an unfused involution has a chief factor of order 2 .
\end{abstract}

\section{Introduction}

Let $G$ be a finite group, $p$ be a prime, and $S$ be a Sylow $p$-subgroup of $G$. For any $x \in G$ and $H \leq G$, let $x^{H}$ denote the set of $H$-conjugates of $x$. We shall say that an element $x \in S$ is unfused in $G$ if $x^{G} \cap S=x^{S}$. (The property of being unfused is easily seen to be independent of the choice of $S$.)

It is a well-known result (an application of the transfer homomorphism) that if all $p$-elements of $G$ are unfused then $G$ is $p$-nilpotent. It seems reasonable, therefore, to ask what consequences might ensue from the existence of a single unfused class of $p$-elements. If $G$ is $p$-solvable, a simple induction argument shows that if $x \in G$ is a non-identity unfused $p$-element, then $G$ has a central chief factor of order $p$ and, in fact, $x \notin[G, x]$. Here, we show that in the case that $x$ is an involution, the same conclusion holds without any solvability hypothesis.

Main Theorem If $G$ is a finite group and $x$ is an unfused involution in $G$, then $x \notin[G, x]$.

If $x$ is an involution, $[G, x]$ consists simply of all products of an even number of conjugates of $x$ and so the conclusion of the theorem is equivalent to the statement that elements of $\left\langle x^{G}\right\rangle$ have a well-defined parity with respect to their representation as products of conjugates of $x$. Indeed, transpositions in symmetric groups of degree $2^{k}$ or $2^{k}+1$ provide perhaps the simplest examples of non-central unfused involutions (Lemma 3.1). Non-trivial examples of unfused involutions may also be found in certain classical matrix groups. For example, if $q \equiv 3(\bmod 4)$, a Sylow 2-subgroup $S$ of $G L_{2}(q)$ is semi-dihedral and the non-central involutions of $S$ are all conjugate in $S$. The same applies to the unitary groups $U_{2}(q)$ if $q \equiv 1(\bmod 4)$. For a projective example, suppose that $q \equiv 1(\bmod 4)$ and $\lambda$ is not a square in the field of order $q$. If $x=\left(\begin{array}{cc}0 & 1 \\ \lambda & 0\end{array}\right)$, then $\bar{x}=x Z\left(G L_{2}(q)\right)$ is an unfused involution in $P G L_{2}(q)$. These examples are, of course, all non-simple, the main thrust of the theorem being that unfused involutions cannot occur in simple groups. (It is perhaps worth noting that simplicity is not precluded by the weaker hypothesis that the involution $x$ of a Sylow 2-subgroup $S$ of $G$ lies outside the subgroup $\left\langle[g, x]: x^{g} \in S\right\rangle[5]$.) 
The statement of the theorem is formally reminiscent of the celebrated $Z^{*}$ theorem of G. Glauberman [6]: If $x \in S \in S y l_{2}(G)$ is an involution for which $x^{G} \cap S=\{x\}$, then $[G, x] \subseteq O_{2^{\prime}}(G)$. However, the similarity may be superficial. Unlike the case with Glauberman's theorem, the result above depends critically on the hypothesis that $x$ is an involution. (For example, if $G$ has dihedral Sylow 2-subgroups, any element of order 4 is obviously unfused.) Moreover, it is not immediately evident that either result is a consequence of the other.

Of course, the significance of the $Z^{*}$-theorem rests largely on the fact that its proof is independent of the classification of finite simple groups (in which it, in fact, plays an important role). Such a proof of our result has eluded us and so we have resorted to a direct assault with the full force of the classification. Needless to say, a classification-free proof would be much more interesting.

The reduction to the case of a simple group and the elimination of the alternating groups as potential counterexamples are each quick and elementary. The groups of Lie type in characteristic 2 are treated using basic properties of parabolics while the exceptional groups in odd characteristic and the sporadic groups are handled using specific structural data from the literature (particularly the Atlas [4]). For the classical groups in odd characteristic, we have not found a comparably efficient approach and it is this case that occupies the bulk of the argument.

\section{The minimal counterexample}

Lemma 2.1 If $H$ is a nilpotent group and $x \in H \backslash\{1\}$, then $x \notin[H, x]$.

Proof. Induction on the nilpotence class of $H$ (applied to $H / Z(H)$ ).

Lemma 2.2 A minimal counterexample $G$ to the Main Theorem is a nonabelian simple group.

Proof. Let $G$ be a counterexample of minimal order with an unfused involution $x$. Since the hypothesis is independent of which of the Sylow 2-subgroups containing $x$ we take for $S$, we may assume that $C_{S}(x)$ is a Sylow 2-subgroup of $C_{G}(x)$.

Let $N$ be a minimal normal subgroup of $G$. If $x \notin N$ then $x N$ is an unfused involution in $G / N$. By the minimality of $G, x N \notin[G / N, x N]=[G, x] N / N$, whence $x \notin[G, x]$, a contradiction. Therefore, $x \in N$.

Let $H=C_{G}(x) N$. Then $C_{S}(x)(S \cap N)$ is a Sylow 2-subgroup of $H$ and so $S \cap H=C_{S}(x)(S \cap N)$. Also, $x$ is unfused in $H$, for if $h \in H$ such that $x^{h} \in S \cap H$ then $x^{h}=x^{s}$ for some $s \in S$ and, because $C_{G}(x) \leq H, s \in S \cap H$.

If $H \neq G$, then by the inductive hypothesis, $x \notin[H, x]=[N, x]$ and so $N$ has a chief factor $\left\langle x^{N}\right\rangle /[N, x] \cong Z_{2}$. We conclude that $N$ is an elementary abelian 2 -group and so $x \in N \leq O_{2}(G)$. But in this case, $x^{G} \subseteq S$, whence $x^{G}=x^{S}$. It follows that $G=C_{G}(x) S$ and so $x \in[G, x]=[S, x]$, contradicting the previous lemma. Therefore, $H=G$ and $S=S \cap H=C_{S}(x)(S \cap N)$.

From this factorization of $S$, it follows that $x$ is unfused in $N$ and so if $N \neq G$, the inductive hypothesis implies that $x \notin[N, x]$. But because $G=C_{G}(x) N$, $[N, x]=[G, x]$ and so this contradicts that fact that $x \in[G, x]$. Therefore, $N=G$ and so $G$ is a simple non-abelian group.

Remark: Although the Main Theorem itself is false for $p>2$, the preceding argument works for all primes $p$ and is easily adapted to prove what was claimed 
in the Introduction, that if $x$ is an unfused $p$-element in a $p$-solvable group $G$, then $x \notin[G, x]$.

Lemma 2.3 Let $x \in G$ be an unfused involution and let $x \in S \in \operatorname{Syl}_{2}(G)$. If $x \in O^{2}(G)$ then $x \in S^{\prime}$. If $x \notin Z^{*}(G)$ then $x \notin Z(S)$. In particular, if both of these hypotheses hold, then $G$ has at least two classes of involutions and $\Omega_{1}\left(S^{\prime}\right) \not Z Z(S)$.

Proof. If $\theta$ is the transfer homomorphism $G \rightarrow S / S^{\prime}$, then because $x$ is unfused in $G, \theta(x)=x^{|G: S|} S^{\prime}=x S^{\prime}$. Since $x \in O^{2}(G) \leq \operatorname{ker} \theta, x \in S^{\prime}$. If $x \in Z(S)$ then $x^{G} \cap S=\{x\}$ and so $x \in Z^{*}(G)$ by Glauberman's theorem.

The following simple observation about a minimal counterexample will prove useful in eliminating certain groups of Lie type and some sporadic groups:

Lemma 2.4 Let $G$ be a minimal counterexample to the Main Theorem and assume that $x \in H<G$ and $|G: H|$ is odd. If $N \unlhd H$ and $(H / N)^{\prime}$ is a direct product of non-abelian simple groups, then $x \in N$.

Proof. If $x \notin N$ then, because $|G: H|$ is odd, $x N$ is an unfused involution in $\bar{H}=H / N$ and so, by the minimality of $G, \bar{x}=x N \notin[\bar{H}, \bar{x}]$. But $x \in S^{\prime}$ by the preceding lemma and so $\bar{x} \in \bar{H}^{\prime}$. Therefore, $\bar{x}[\bar{H}, \bar{x}]$ generates a chief factor of order 2 of $\bar{H}^{\prime}$. But by hypothesis, $\bar{H}^{\prime}$ has no such chief factor and so $x \in N$.

Corollary 2.5 Let $G$ be a minimal counterexample to the Main Theorem and assume that $S \in \mathrm{Syl}_{2}(G)$ such that $S \leq M<G$ and $\left(M / O_{2,2^{\prime}}(M)\right)^{\prime}$ is a direct product of non-abelian simple groups. Then $M \leq N_{G}\left(\left\langle x^{S}\right\rangle\right)$.

Proof. By Lemma 2.4, $x \in O_{2,2^{\prime}}(M)$ and so $x \in O_{2}(M)$, whence $x^{M}=x^{S}$ and in particular, $\left\langle x^{S}\right\rangle \unlhd M$.

\section{The alternating groups}

Lemma 3.1 If $x$ is an involution in the symmetric group $S_{n}$, then $x$ is unfused in $S_{n}$ if and only if $x$ is a transposition and $n=2^{k}$ or $2^{k}+1$ for some $k$.

Proof. We may assume without loss that $x=(12)(34) \ldots(2 r-12 r) \in\langle X\rangle$ where $2 r \leq m=2\left[\frac{n}{2}\right]$ and $X=\{(12),(34), \ldots,(m-1 m)\}$. Suppose that $r \geq 2$. If $y=x^{(23)}=(13)(24)(56) \ldots(2 r-12 r)$, conjugation of $X$ by $y$ interchanges (12) and (34) and fixes all other elements of $X$. In particular, $y \in N_{G}(\langle X\rangle)$ and so $\langle X, y\rangle \leq S \in S y l_{2}(G)$. $X$ is a maximal set of pairwise disjoint transpositions in $S_{n}$ and so it is precisely the set of transpositions in $S$. Thus, $\langle X\rangle \unlhd S$ and so $x^{S} \subseteq\langle X\rangle$. Then $y=x^{(23)} \in x^{S_{n}} \cap S=x^{S} \subseteq\langle X\rangle$, a contradiction because $y$ does not centralize (12). We conclude that $r=1$ and so $x$ is a transposition.

If $n=2^{n_{1}}+2^{n_{2}}+\cdots+2^{n_{t}}\left(0 \leq n_{1}<n_{2}<\ldots<n_{t}\right)$ is the binary representation of $n$, then any partition of $\{1,2, \ldots, n\}$ into $t$ subsets of cardinality $2^{n_{i}}$, $1 \leq i \leq t$, defines an embedding of $S_{2^{n_{1}}} \times S_{2^{n_{2}}} \times \ldots \times S_{2^{n_{t}}}$ as a subgroup of odd index in $S_{n}$. Hence, a Sylow 2-subgroup $S$ of $S_{n}$ is a direct product of Sylow 2-subgroups of the $S_{2^{n_{i}}}$. Transpositions in distinct direct factors of $S$ are not $S$-conjugate and so if some transposition of $S_{n}$ is unfused, $S$ must be directly indecomposable. Therefore, $n=2^{k}$ or $2^{k}+1$ for some $k$. 
If $n$ is of the form $2^{k}$ or $2^{k}+1$ then, because $S \cong\left(\mathbf{Z}_{2} \prec \mathbf{Z}_{2}\right) \prec \ldots \curlywedge \mathbf{Z}_{2}$, the elements of $X$ (i.e. the transpositions in $S$ ) are all $S$-conjugate (and hence, $S$-conjugate to $x$ ) and so all transpositions are unfused in $S_{n}$.

Corollary 3.2 The alternating groups contain no unfused involutions.

Proof. Suppose that $x$ is an unfused involution in $A_{n}$ and $x \in T \in S y l_{2}\left(A_{n}\right)$. Let $T \leq S \in S y l_{2}\left(S_{n}\right)$ and let $y \in S \backslash T$, so $S_{n}=A_{n}\langle y\rangle$ and $S=T\langle y\rangle$. Then $x^{A_{n}} \cap S=x^{A_{n}} \cap S \cap A_{n}=x^{A_{n}} \cap T \subseteq x^{T}$ and so $x^{A_{n} y} \cap S=\left(x^{A_{n}} \cap S\right)^{y} \subseteq x^{T y} \subseteq x^{S}$. Thus, $x^{S_{n}} \cap S \subseteq x^{S}$ and so $x$ is unfused in $S_{n}$, contradicting the preceding lemma.

\section{The classical groups in odd characteristic}

In this section, $V$ is a vector space of dimension $n \geq 2$ over a finite field $F$ of odd order and ( , ) denotes a binary form on $V$ which is either the zero form or a non-degenerate alternating, hermitian or symmetric form. In the hermitian case, $|F|=q^{2}$ and the form is sesquilinear with respect to the Frobenius automorphism $c \mapsto c^{q}$. In all other cases, $|F|=q$. We shall use the symbol "ㅇ" to denote both isometric spaces and isomorphic groups. By a "direct sum", we shall always mean an orthogonal direct sum.

It is a standard fact that if $n=2$, then in the alternating and hermitian cases, $V$ is a hyperbolic plane (i.e. $V$ is generated by isotropic vectors $u$ and $v$ with $(u, v)=1)$. In the symmetric case, it is either a hyperbolic plane, denoted here by $\Pi^{+}$, or what we shall call an elliptic (totally anisotropic) plane $\Pi^{-}$. (See, for example, [9].)

$G$ will denote the group of isometries of $V$ with respect to the form (i.e. the general linear, symplectic, unitary, or an orthogonal group on $V$ ).

Recall the following order formulae (for odd $q$ ):

A. If $G=G L_{n}(q)$, then

$$
|G|=q^{n(n-1)} \prod_{i=1}^{n}\left(q^{i}-1\right) .
$$

B. If $G=S p_{n}(q)$, then $n=2 m$ and

$$
|G|=q^{m^{2}} \prod_{i=1}^{m}\left(q^{2 i}-1\right) .
$$

C. If $G=U_{n}(q)$, then

$$
|G|=q^{n(n-1) / 2} \prod_{i=1}^{n}\left(q^{i}-(-1)^{i}\right) .
$$

D. If $G$ is an orthogonal group, then one of the following holds: 
1. $n=2 m+1, G=O_{n}(q)$ and

$$
|G|=2 q^{m^{2}} \prod_{i=1}^{m}\left(q^{2 i}-1\right) .
$$

2. $n=2 m, G=O_{n}^{\eta}(q)$ (where $\eta= \pm 1$ ) and

$$
|G|=2 q^{m(m-1)}\left(q^{m}-\eta\right) \prod_{i=1}^{m-1}\left(q^{2 i}-1\right) .
$$

We assume that the element $x \in G^{\prime}$ satisfies $x^{2}=\lambda I \in Z(G)$ for some $\lambda \in F$. The goal of the section is to show that the involution $x Z(G)$ is not unfused in $(G / Z(G))^{\prime}$ (so a counterexample to the Main Theorem is not to be found among the classical groups of odd characteristic).

In the orthogonal case, we define $\epsilon= \pm 1$ by the congruence $q \equiv \epsilon(\bmod 4)$. Thus, $\epsilon=1$ or -1 according as whether or not $-1 \in F^{2}=\left\{c^{2}: c \in F\right\}$.

Lemma 4.1 Assume that the form ( , ) is non-zero.

If $V$ is a symplectic or unitary space and $\operatorname{dim} V \geq 2$, then $V$ contains an $x$-invariant hyperbolic plane.

If $V$ is an orthogonal space and $\operatorname{dim} V \geq 3$, then $V$ contains an $x$-invariant plane isometric to $\Pi^{\epsilon}$.

Proof. In the symplectic and unitary cases, non-degenerate planes are hyperbolic and so for the first statement, it is enough to show that if $\operatorname{dim} V \geq 3$, then $V$ contains a non-degenerate $x$-invariant plane. Suppose that $\lambda=\mu^{2}$ for some $\mu \in F$. Then $\pm \mu$ are the eigenvalues of $x$ and since both lie in $F, V$ is spanned by eigenvectors of $x$. Because $V$ is non-degenerate, there exists a pair of (not necessarily distinct) eigenvectors $u, v$ such that $(u, v) \neq 0$. If one of these vectors, say $u$, is anisotropic, then $V=L \perp L^{\perp}$ where $L=\langle u\rangle$ and, by induction on $\operatorname{dim} V, L^{\perp}$ contains an $x$-invariant hyperbolic plane. But if $u$ and $v$ are isotropic then $\langle u, v\rangle$ is an $x$-invariant hyperbolic plane. Therefore, we may assume that $\lambda \notin\left(F^{\times}\right)^{2}$ (and in particular, $\lambda \neq 1$ ), whence $V$ has no $x$-invariant one-dimensional subspaces. Then if $v \in V$ and the $x$ invariant plane $L=\left\langle v, v^{x}\right\rangle$ is degenerate, $L \cap L^{\perp}$ is non-zero and $x$-invariant, whence $L \cap L^{\perp}=L$. Hence, we may assume that $(v, v)=\left(v, v^{x}\right)=0$ for all $v \in V$ (and so $V$ is a symplectic space). But then for any $u, v \in V$, $0=\left(u+v^{x},\left(u+v^{x}\right)^{x}\right)=\left(u+v^{x}, u^{x}+\lambda v\right)=(\lambda-1)(u, v)$ and so $(u, v)=0$, contradicting our assumption that the form $(, \quad)$ is non-zero.

For the second statement, we show that there exist $u, v \in V$ such that $\langle u, v\rangle$ is $x$-invariant, $(u, u)=(v, v) \neq 0$, and $(u, v)=0$. This suffices because if $\alpha, \beta \in F, \alpha u+\beta v \in\langle u, v\rangle$ is isotropic if and only if $\alpha^{2}+\beta^{2}=0$. But such a pair $\alpha, \beta$ exists if and only if $-1 \in F^{2}$ (i.e. $\epsilon=1$ ). Therefore, because $\langle u, v\rangle$ is non-degenerate, it is isometric to $\Pi^{+}$if $\epsilon=1$ and to $\Pi^{-}$if $\epsilon=-1$.

If $\lambda \neq 1$, let $v \in V$ be anisotropic and let $u=v^{x}$. Then $\langle u, v\rangle$ is $x$-invariant, $(u, u)=(v, v)$ and $(u, v)=\left(u^{x}, v^{x}\right)=(\lambda v, u)=\lambda(u, v)$, whence $(u, v)=0$.

If $\lambda=1$, then $x$ has eigenvalues \pm 1 and $V=E_{1} \perp E_{-1}$, where $E_{ \pm 1}$ are the corresponding eigenspaces. Because $\operatorname{dim} V \geq 3, V$ contains three pairwise orthogonal anisotropic eigenvectors, say $v, u_{1}$ and $u_{2}$. There exist $\alpha, \beta \in F$ such that $\alpha^{2}\left(u_{1}, u_{1}\right)+\beta^{2}\left(u_{2}, u_{2}\right)=(v, v)\left([9]\right.$, Lemma 11.1) and so, if $u=\alpha u_{1}+\beta u_{2}$, 
$(u, u)=(v, v)$ and the pair $u, v$ satisfies our requirements.

Lemma 4.2 There exists a decomposition $V=V_{1} \perp \ldots \perp V_{m} \perp U$ such that each summand is $x$-invariant, $\operatorname{dim} V_{i}=2$ for all $i$ and $\operatorname{dim} U \leq 1$. Moreover, in the orthogonal case, $V_{i} \cong \Pi^{\epsilon}$ for $1 \leq i \leq m-1$ and if $U \neq 0, V_{m} \cong \Pi^{\epsilon}$.

Proof. We may assume that $\operatorname{dim} V \geq 3$. (Note that the second statement is vacuous if $\operatorname{dim} V \leq 2$ ). The general linear case is immediate from Maschke's theorem. In the other cases, because $V=W \perp W^{\perp}$ for any non-degenerate subspace $W$ of $V$, we apply the preceding lemma and induction on $\operatorname{dim} V$.

Lemma 4.3 Let $H$ be the (setwise) stabilizer in $G$ of $\left\{V_{1}, \ldots, V_{m}, U\right\}$.

If $G$ is a general linear group, $H \cong G L_{2}(q)$ 乙 $S_{m}$ or $\left(G L_{2}(q) \curlywedge S_{m}\right) \times G L_{1}(q)$, according as $\operatorname{dim} U=0$ or 1 .

If $G$ is a symplectic group, then $H \cong S p_{2}(q)$ < $S_{m}$.

If $G$ is a unitary group, then $H \cong U_{2}(q) \curlywedge S_{m}$ or $U_{2}(q) \curlywedge S_{m} \times U_{1}(q)$, according as $\operatorname{dim} U=0$ or 1 .

If $G$ is an orthogonal group and if $\operatorname{dim} U=1$, then $H \cong\left(O_{2}^{\epsilon}(q)\right.$ ᄀ $\left.S_{m}\right) \times O_{1}(q)$.

If $G$ is an orthogonal group and if $\operatorname{dim} U=0$, then $H \cong O_{2}^{\epsilon}(q)<S_{m}$ or $\left(O_{2}^{\epsilon}(q) \prec S_{m-1}\right) \times O_{2}^{-\epsilon}(q)$, according as $V_{m}$ is isometric to $\Pi^{\epsilon}$ or $\Pi^{-\epsilon}$.

In all cases, $|G: H|$ is odd.

Proof. All of the statements except the last are a consequence of Lemma 4.2. The last statement follows from the order formulas. In verifying this, it may be useful to note that if $i_{2}$ denotes the 2-part of the integer $i$, then

(i) if $i$ is odd, $\left(q^{i} \pm 1\right)_{2}=(q \pm 1)_{2}$, and

(ii) if $i$ is even, $\left(q^{i}-1\right)_{2}=i_{2}(q-\epsilon)_{2}$.

Note that Lemma 4.3 provides a concise description of the Sylow 2-subgroups of each of the classical groups in odd characteristic. For the orthogonal groups, this description seems somewhat more transparent than that given in [3].

While our concern is with unfused involutions in $(G / Z(G))^{\prime}$, the upshot of the next observation is that it is sufficient to consider involution fusion in $G / Z(G)$ (whose Sylow 2-subgroups are, in view of the preceding lemma, more conveniently described).

Corollary 4.4 If $\bar{G}=G / Z(G)$ and $\bar{x}=x Z(G)$ is an unfused involution in $\bar{G}^{\prime}$, then $\bar{x}$ is unfused in $\bar{G}$.

Proof. We claim first that $G /\left(C_{G}(x) G^{\prime}\right)$ is an abelian 2-group. If $G_{0}=\{g \in$ $G: \operatorname{det} g=1\}$ then $G^{\prime}=G_{0}$ except in the orthogonal case when $\left|G_{0}: G^{\prime}\right|=2$ ([9], Chapter 11). Thus, it suffices to show that $G /\left(C_{G}(x) G_{0}\right)$ is a 2-group.

By Lemma 4.3, we may let $V=V_{1} \perp W$ where $\operatorname{dim} V_{1}=2$ and both summands are $x$-invariant. If $g \in G$, let $h \in G$ be the transformation of $V$ which induces scalar multiplication by $\operatorname{det} g$ on $V_{1}$ and fixes each element of $W$. Since $V_{1}$ and $W$ are $x$-invariant, $h \in C_{G}(x)$. But $\operatorname{det} g^{2}=\operatorname{det} h$ and so $g^{2} \in h G_{0} \subseteq C_{G}(x) G_{0}$. Therefore, $G /\left(C_{G}(x) G_{0}\right)$ has exponent at most 2 and the claim is proved.

It follows that if $S \in S y l_{2}(G)$, then $G=C_{G}(x) G^{\prime} S$. Assuming that $\bar{x} \in \bar{S}=$ $S Z(G) / Z(G)$, then because $\bar{x}$ is unfused in $\overline{G^{\prime}}$,

$$
\bar{x}^{\bar{G}} \cap \bar{S}=\bar{x}^{\overline{G^{\prime} S}} \cap \bar{S}=\left(\bar{x}^{\bar{G}^{\prime}} \cap \bar{S}\right)^{\bar{S}}=\left(\bar{x}^{\left(\overline{S \cap G^{\prime}}\right)}\right)^{\bar{S}}=\bar{x}^{\bar{S}}
$$


and so $\bar{x}$ is unfused in $\bar{G}$.

Lemma 4.5 Let $X=V_{1} \perp V_{2}$ be a subspace of $V$, where $V_{1}$ and $V_{2}$ are nondegenerate $x$-invariant planes. In the orthogonal case, assume that $V_{i} \cong \Pi^{\epsilon}$ for $i=1,2$. If no eigenspace of $x$ in $X$ has dimension greater than 2, then either

(a) there is a subspace $W$ of $X$ such that $W \cong V_{1} \cong V_{2}$ and $X=W \perp W^{x}$ or

(b) $x^{2}=1$ (whence, $X$ admits a decomposition into eigenspaces $E_{1} \perp E_{-1}$ ), $E_{1} \cong E_{-1} \cong \Pi^{-\epsilon}$ and $V_{1} \cap E_{1} \cong V_{1} \cap E_{-1} ¥ V_{2} \cap E_{1} \cong V_{2} \cap E_{-1}$.

Proof. Suppose first that $x$ has an eigenvector in $X$ so $X$ is a sum (not necessarily orthogonal) of eigenspaces $E_{\mu}$ and $E_{-\mu}$ (and each $V_{i}$ is a sum of $V_{i} \cap E_{\mu}$ and $\left.V_{i} \cap E_{-\mu}\right)$, where $\mu^{2}=\lambda$.

If $u, v \in E_{\mu},(u, v)=\left(u^{x}, v^{x}\right)=(\mu u, \mu v)=\mu^{1+q}(u, v)$ so either $\mu^{1+q}=1$ or $E_{\mu}$ is totally isotropic. On the other hand, if $u \in E_{\mu}$ and $v \in E_{-\mu}$ then $(u, v)=\left(u^{x}, v^{x}\right)=(\mu u,-\mu v)=-\mu^{1+q}(u, v)$ so either $\mu^{1+q}=-1$ or $E_{\mu} \perp E_{-\mu}$. Thus, either $X=E_{\mu} \perp E_{-\mu}$ or each $E_{ \pm \mu}$ is totally isotropic.

In the former case, $\operatorname{dim} E_{\mu}=\operatorname{dim} E_{-\mu}=2$. Then $E_{\mu} \cong E_{-\mu}$ (in the orthogonal case, because $\Pi^{\epsilon} \perp \Pi^{\epsilon} \not \Pi^{\epsilon} \perp \Pi^{-\epsilon}$ by the Witt Cancellation theorem) so we may choose bases $\left\{r_{1}, r_{2}\right\}$ for $E_{\mu}$ and $\left\{s_{1}, s_{2}\right\}$ for $E_{-\mu}$ such that $\left(r_{i}, r_{j}\right)=\left(s_{i}, s_{j}\right)$ for $i, j \in\{1,2\}$. Let $w_{1}=r_{1}+s_{1}, w_{2}=r_{2}+s_{2}$ and $W=\left\langle w_{1}, w_{2}\right\rangle$. Then $X=W \perp W^{x}$. If the form on $X$ is zero, symplectic or unitary, we are done since $W \cong V_{1} \cong V_{2}$ and so assume that $X$ is an orthogonal space.

Then $\mu= \pm 1$ (and so $x^{2}=1$ ) and $\left(w_{i}, w_{j}\right)=\left(r_{i}+s_{i}, r_{j}+s_{j}\right)=\left(r_{i}, r_{j}\right)+$ $\left(s_{i}, s_{j}\right)=2\left(r_{i}, r_{j}\right)$. It follows that $W$ contains an isotropic vector if and only if $E_{1}$ does and so $W \cong E_{1} \cong E_{-1}$. If $E_{1} \cong \Pi^{\epsilon}$, then (a) holds and so assume that $E_{1} \cong \Pi^{-\epsilon}$. We claim in this case that (b) holds.

$V_{1} \cap E_{1}$ and $V_{1} \cap E_{-1}$ are each 1-dimensional so let $\left\{e_{1}\right\}$ and $\left\{f_{1}\right\}$ be bases for these respective subspaces. Suppose that $\left(f_{1}, f_{1}\right) /\left(e_{1}, e_{1}\right) \notin\left(F^{\times}\right)^{2}$. Then $V_{1} \cong \Pi^{+}$(that is, it contains an isotropic vector $\left.x e_{1}+y f_{1}\right)$ if and only if $-\left(f_{1}, f_{1}\right) /\left(e_{1}, e_{1}\right) \in\left(F^{\times}\right)^{2}$. But this, in turn, holds if and only if $-1 \notin\left(F^{\times}\right)^{2}$ (and so $\epsilon=-1$ ), contradicting the hypothesis that $V_{1} \cong \Pi^{\epsilon}$. Therefore, $\left(f_{1}, f_{1}\right) /\left(e_{1}, e_{1}\right) \in F^{2}$ and so $V_{1} \cap E_{1} \cong V_{1} \cap E_{-1}$. Similarly, $V_{2} \cap E_{1} \cong V_{2} \cap E_{-1}$.

Suppose that $V_{1} \cap E_{1} \cong V_{2} \cap E_{1}$. Then we may choose bases $\left\{e_{1}\right\}$ and $\left\{d_{1}\right\}$ for these respective spaces so that $\left(e_{1}, e_{1}\right)=\left(d_{1}, d_{1}\right)$. Hence, $\left(x e_{1}+y d_{1}, x e_{1}+y d_{1}\right)=$ $\left(x^{2}+y^{2}\right)\left(e_{1}, e_{1}\right)$ and so $E_{1}=\left\langle e_{1}, d_{1}\right\rangle \cong \Pi^{+}$if and only if $-1 \in\left(F^{\times}\right)^{2}$ (or equivalently, $\epsilon=1)$. But $E_{1} \cong \Pi^{-\epsilon}$, a contradiction. Therefore, $V_{1} \cap E_{1} \neq$ $V_{2} \cap E_{1}$ and (b) holds as claimed. This completes the proof in the case that $E_{\mu} \perp E_{-\mu}$.

Suppose that $E_{\mu}$ and $E_{-\mu}$ are totally isotropic, whence $V_{1}$ and $V_{2}$ each contain isotropic vectors and so are hyperbolic planes. Let $V_{i} \cap E_{\mu}=\left\langle r_{i}\right\rangle$, $V_{i} \cap E_{-\mu}=\left\langle s_{i}\right\rangle, i=1,2$ and define $w_{1}=r_{1}+s_{2}, w_{2}=r_{2}+s_{1}$ and $W=\left\langle w_{1}, w_{2}\right\rangle$. Then $X=W \perp W^{\perp}$. Also, $\left(w_{1}, w_{1}\right)=\left(r_{1}+s_{2}, r_{1}+s_{2}\right)=0$ and so $W$ is also a hyperbolic plane. Thus, $W \cong V_{1} \cong V_{2}$ and (a) holds.

We are left with the case that $x$ has no eigenvectors in $X$ (whence $\lambda \neq 1$ ). In this case, let $v_{i} \in V_{i}, i=1,2$, such that $\left(v_{1}, v_{1}\right)=\left(v_{2}, v_{2}\right)$ and let $u_{i}=v_{i}^{x}$, so $\left\langle v_{i}, u_{i}\right\rangle=V_{i}$ and $\left(u_{1}, u_{1}\right)=\left(u_{2}, u_{2}\right)$.

In the general linear case, we may simply take $W=\left\langle v_{1}, v_{2}\right\rangle$.

If $X$ is a symplectic space, $\left(v_{i}, u_{i}\right) \neq 0$ (because $V_{i}$ is non-degenerate) and $\left(v_{i}, u_{i}\right)=\left(v_{i}^{x}, u_{i}^{x}\right)=\left(u_{i}, \lambda v_{i}\right)=-\lambda\left(v_{i}, u_{i}\right)$ so $\lambda=-1$. Choose $\alpha, \beta \in F_{q}$ such 
that $\alpha^{2}+\beta^{2}=-\left(v_{2}, u_{2}\right) /\left(v_{1}, u_{1}\right)$ and let $w_{1}=\alpha v_{1}+\beta u_{1}+v_{2}, w_{2}=-\beta v_{1}+$ $\alpha u_{1}-u_{2}$ and $W=\left\langle w_{1}, w_{2}\right\rangle$. W is then a hyperbolic plane and $X=W \perp W^{x}$.

If $X$ is a unitary space, choose $v_{i}$ to be isotropic so $\left(v_{i}, u_{i}\right) \neq 0$ (since $\left.\left\langle v_{i}, u_{i}\right\rangle=V_{i}\right)$. Then we have $\left(v_{i}, u_{i}\right)=\left(v_{i}^{x}, u_{i}^{x}\right)=\left(u_{i}, \lambda v_{i}\right)=\lambda^{q}\left(v_{i}, u_{i}\right)^{q}$ and so $\left(v_{1}, u_{1}\right)^{q-1}=\lambda^{-q}=\left(v_{2}, u_{2}\right)^{q-1}$. Therefore, $\left(\left(v_{2}, u_{2}\right) /\left(v_{1}, u_{1}\right)\right)^{q-1}=1$, whence $\left(v_{2}, u_{2}\right) /\left(v_{1}, u_{1}\right) \in F_{q}$ and so $c^{q+1}=-\left(v_{2}, u_{2}\right) /\left(v_{1}, u_{1}\right)$ for some $c \in F$. If $w_{1}=c v_{1}+v_{2}$ and $w_{2}=c u_{1}-u_{2}$ then $w_{1}$ and $w_{2}$ are isotropic and $\left(w_{1}, w_{2}\right) \neq 0$, so $W=\left\langle w_{1}, w_{2}\right\rangle$ is a hyperbolic plane. Moreover, $w_{1}^{x}=c u_{1}+u_{2} \in W^{\perp}$ and $w_{2}^{x}=\lambda\left(c v_{1}-v_{2}\right) \in W^{\perp}$. Therefore, $W^{x}=W^{\perp}$ and $X=W \perp W^{x}$.

Finally, suppose that $X$ is an orthogonal space. Then $\left(v, v^{x}\right)=\left(v^{x}, v^{x^{2}}\right)=$ $\left(v^{x}, \lambda v\right)=\lambda\left(v, v^{x}\right)$, whence (since $\left.\lambda \neq 1\right),\left(v, v^{x}\right)=0$ for all $v \in X$ (and in particular, $\left(v_{i}, u_{i}\right)=0$ for $\left.i=1,2\right)$. If $V_{i}$ contained an isotropic vector $v$, then $V_{i}=\left\langle v, v^{x}\right\rangle$ would be totally isotropic, contradicting the non-degeneracy of $X$. Hence, each $V_{i}$ is an elliptic plane $\Pi^{-}$and so, $\epsilon=-1$. If $W=\left\langle v_{1}, v_{2}\right\rangle$, then $X=W \perp W^{x}$. Also, $\left(\alpha v_{1}+\beta v_{2}, \alpha v_{1}+\beta v_{2}\right)=\left(\alpha^{2}+\beta^{2}\right)\left(v_{1}, v_{1}\right)$ and since $\epsilon=-1, \alpha^{2}+\beta^{2} \neq 0$ for all $\alpha, \beta \in F$. Therefore, $W \cong \Pi^{-}$and the proof is complete.

Lemma 4.6 Let $H=H_{1} \times H_{2}$ be a subgroup of odd index in $G$ and assume that $x \in H$ such that $x Z(G)$ is an unfused involution in $G / Z(G)$. If $x=x_{1} x_{2}$, where $x_{i} \in H_{i}, i=1,2$, then for each $i, x_{i} Z\left(H_{i}\right)$ is unfused in $H_{i} / Z\left(H_{i}\right)$.

Proof. We may assume that $x_{i} \in S_{i} \in S y l_{2}\left(H_{i}\right)$ for $i=1,2$. If $h_{1} \in H_{1}$ such that $x_{1}^{h_{1}} \in S_{1} Z\left(H_{1}\right)$ then $x_{1}^{h_{1}} \in S_{1}$ and so $x^{h_{1}}=x_{1}^{h_{1}} x_{2} \in S=S_{1} \times S_{2} \in \operatorname{Syl}_{2}(G)$. By hypothesis, there exist elements $s_{i} \in S_{i}, i=1,2$, such that

$$
x_{1}^{h_{1}} x_{2} Z(G)=x^{s_{1} s_{2}} Z(G)=x_{1}^{s_{1}} x_{2}^{s_{2}} Z(G) .
$$

Thus, for some $z \in Z(G), x_{1}^{-s_{1}} x_{1}^{h_{1}}=x_{2}^{s_{2}} x_{2}^{-1} z \in H_{1} \cap C_{G}\left(H_{1}\right)=Z\left(H_{1}\right)$ and so $x_{1}^{h_{1}} Z\left(H_{1}\right)=x_{1}^{s_{1}} Z\left(H_{1}\right)$ as required. The proof for $H_{2} / Z\left(H_{2}\right)$ is similar.

Theorem 1 Let $G$ be a classical group over a field $F$ of odd order and let $V$ be the natural FG-module with associated $G$-invariant form (, ). Assume in the orthogonal case that $\operatorname{dim} V \geq 3$; otherwise, that $\operatorname{dim} V \geq 2$. If $\bar{x}=x Z(G)$ is an unfused involution in $\bar{G}=G / Z(G)$, then $\bar{x} \notin \bar{G}^{\prime}$.

Proof. Assume that the pair $(G, V)$ is a counterexample with $V$ of minimal $F$-dimension, where $|F|=q^{2}$ in the unitary case and $|F|=q$ otherwise.

Suppose first that $\operatorname{dim} V=2$ and $G \cong P G L_{2}(q)$. Because $P S L_{2}(q)=$ $O^{2}\left(P S L_{2}(q)\right)$ has dihedral Sylow 2-subgroups, it has a unique conjugacy class of involutions (by the Thompson transfer lemma) and so from the hypothesis, every involution of $P S L_{2}(q)$ is unfused in $P G L_{2}(q)$. But if $\bar{T} \in S y l_{2}\left(P S L_{2}(q)\right)$, $\bar{T} \unlhd \bar{S}$ for some $\bar{S} \in S y l_{2}\left(P G L_{2}(q)\right)$, whence $Z(\bar{T})^{\bar{S}}=Z(\bar{T})$. The unfused hypothesis then implies that all involutions of $\bar{T}$ lie in $Z(\bar{T})$, which is false. Therefore, $G \neq P G L_{2}(q)$. Because $S p_{2}(q) \cong S L_{2}(q) \cong S U_{2}(q)$ ([9], 8.1 and 10.9), similar considerations eliminate the two-dimensional symplectic and unitary cases. Thus, in the non-orthogonal cases, we may assume that $\operatorname{dim} V \geq 3$.

Because $P \Omega_{3}(q) \cong P S L_{2}(q)([9], 11.8)$ and $P \Omega_{4}^{-}(q) \cong P S L_{2}\left(q^{2}\right)([9], 12.43)$, the same argument shows that $G$ is not $O_{3}(q)$ or $O_{4}^{-}(q)$. Also $P \Omega_{4}^{+}(q) \cong$ $P S L_{2}(q) \times P S L_{2}(q)([9], 12.39)$ and so $P \Omega_{4}^{+}(q)$ has three classes of involutions, and for a given Sylow 2-subgroup $S$ of $G$, each class contains elements 
of both $Z(S)$ and $S \backslash Z(S)$. Thus, a similar argument eliminates $O_{4}^{+}(q)$ as a possibility for $G$. Therefore, we may assume that $\operatorname{dim} V \geq 5$ in the orthogonal cases.

Let $x \in G^{\prime}$ such that $\bar{x}=x Z(G)$ is an unfused involution in $\bar{G}=G / Z(G)$ (so $x^{2}=\lambda I$ for some $\lambda \in F$ ) and, as in Lemma 4.2, let $V=V_{1} \perp \ldots \perp V_{m} \perp$ $U$. By Lemma 4.3, we may assume that $S \in S y l_{2}(G)$ leaves invariant the set $\left\{V_{1}, \ldots, V_{m}, U\right\}$ in $G$. Because $\bar{G}^{\prime}=O^{2}\left(\bar{G}^{\prime}\right), \bar{x} \in O^{2}(\bar{G})$ and so Lemma 2.3 yields that $\bar{x} \in \bar{S}^{\prime}$. Thus, $x \in S^{\prime} Z(G)$ and so we may assume that $x \in S^{\prime}$ (and $\lambda$ is a 2-element of $F^{\times}$). In the case that $U \neq 0$, elements of $S$ obviously leave $U$ invariant and in the even-dimensional orthogonal case in which $V_{m} \cong \Pi^{-\epsilon}$, elements of $S$ leave $V_{m}$ invariant. We argue now that neither of these cases applies to $V$.

For any subspace $X$ of $V$, let $G_{X}$ denote the subgroup of those elements of $G$ which leave $X$ invariant and induce the identity map on $X^{\perp}$. (Thus, $G_{X}$ is isomorphic to the isometry group of $X$ with respect to the form induced from $V$.) If $U \neq 0$, let $Z=V_{1} \perp \ldots \perp V_{m}$ (so $U=Z^{\perp}$ ). If $V$ is even-dimensional orthogonal and $V_{m} \cong \Pi^{-\epsilon}$, let $Z=V_{1} \perp \ldots, \perp V_{m-1}$ (so $V_{m}=Z^{\perp}$ ). In either case, $S \leq G_{Z} \times G_{Z^{\perp}}$ and so $S=S_{Z} \times S_{Z^{\perp}}$, where $S_{Z} \in S y l_{2}\left(G_{Z}\right)$ and $S_{Z^{\perp}} \in S y l_{2}\left(G_{Z^{\perp}}\right)$. Because $x \in S^{\prime}, x=y z$ where $y \in\left(S_{Z}\right)^{\prime}$ and $z \in\left(S_{Z^{\perp}}\right)^{\prime}$. By Lemma 4.6 , the element $y Z\left(G_{Z}\right) \in\left(G_{Z} / Z\left(G_{Z}\right)\right)^{\prime}$ is unfused in $G_{Z} / Z\left(G_{Z}\right)$ and so, since $2 \leq \operatorname{dim} Z<\operatorname{dim} V$ in the non-orthogonal cases and $3 \leq \operatorname{dim} Z<$ $\operatorname{dim} V$ in the orthogonal case, the minimality of $\operatorname{dim} V$ yields that $y \in Z\left(G_{Z}\right)$. Therefore, $y$ induces on $Z$ scalar multiplication by some $\mu \in F$ and since $y^{2}$ induces scalar multiplication by $\lambda, \mu^{2}=\lambda$. If $Z^{\perp}=U$, then $\left(G_{Z^{\perp}}\right)^{\prime}=1$ and so $z=1$. If $V$ is an even-dimensional orthogonal space and $Z^{\perp}=V_{m}$, then $Z\left(G_{Z}\right)=\{ \pm I\}$. Therefore, $\mu= \pm 1$ and so $\lambda=1$ and $x, y$ and $z$ are all involutions. Because $G_{Z^{\perp}} \cong O_{2}^{-\epsilon}(q)$ is dihedral, $\left(G_{Z^{\perp}}\right)^{\prime}$ is cyclic and so $z \in Z\left(G_{Z^{\perp}}\right)$. In either case, we conclude that $x \in Z(S)$, whence, by Lemma $2.3, x \in Z^{*}(G)$. But $Z^{*}(G)=Z(G)$, contradicting the assumption that $x Z(G)$ is an involution. This proves the assertion of the preceding paragraph, that $U=0$ and, in the orthogonal case, the $V_{k}$ 's are all isometric to $\Pi^{\epsilon}$.

Suppose that for some pair $i, j$, conclusion (a) of Lemma 4.5 holds for the subspace $V_{i} \perp V_{j}$. Thus, assuming without loss that $i=1$ and $j=2, V_{1} \perp V_{2}$ contains a subspace $W \cong V_{1} \cong V_{2}$ such that $V_{1} \perp V_{2}=W \perp W^{x}$. Let $\eta \in S$ be an isometry of $V$ of order 2 which interchanges $V_{1}$ and $V_{2}$ and fixes (pointwise) all $V_{k}$ for $k \geq 3$. Let $\theta: W \rightarrow V_{1}$ be an isometry. Then there is a unique isometry $g$ of $V$ such that for any $w \in W, w^{g}=w^{\theta},\left(w^{x}\right)^{g}=w^{\theta \eta}$ and $v_{k}^{g}=v_{k}$ if $v_{k} \in V_{k}, k \geq 3$. Hence, $v_{1}^{x^{g}}=v_{1}^{g^{-1} x g}=v_{1}^{\eta}$ if $v_{1} \in V_{1}, v_{2}^{x^{g}}=\lambda v_{2}^{\eta^{-1}}=\lambda v_{2}^{\eta}$ if $v_{2} \in V_{2}$, and $v_{k}^{x^{g}}=v_{k}=v_{k}^{\eta}$ if $v_{k} \in V_{k}, k \geq 3$. Therefore, $x^{g} \eta^{-1}$ induces scalar multiplication by $\lambda$ on $V_{2}$ and the identity map on $V_{k}$ for $k \neq 2$. Since $\lambda$ is a 2-element of $F^{\times}$, it follows that $x^{g} \eta^{-1} \in S$, whence $x^{g} \in S$.

By hypothesis, $x^{g}=x^{s} z$ for some $s \in S, z \in Z(G)$. But whereas $x^{g}$ interchanges $V_{1}$ and $V_{2}, x^{s}$ leaves $V_{1}$ and $V_{2}$ invariant (because $x$ leaves all summands invariant and $s$ permutes them) and so we have a contradiction. Thus, conclusion (a) of Lemma 4.5 cannot hold in our counterexample.

Assume that condition (b) of Lemma 4.5 does not hold for any pair $V_{i}, V_{j}, i \neq$ $j$. By Lemma 4.5, for every distinct pair $i, j, x$ has an eigenspace of dimension at least 3 on $V_{i} \perp V_{j}$ and so $x$ induces a scalar transformation on all but at most one of the $V_{k}$ 's. Indeed, $x$ induces the same scalar transformation on $V_{k}$ for all $k \geq 2$ and has one-dimensional eigenspaces in $V_{1}$. We write $V_{1}=\left\langle u_{1}, v_{1}\right\rangle$ 
where $u_{1}^{x}=-\mu u_{1}$ and $v_{1}^{x}=\mu v_{1}$ for some $\mu \in F$ (with $\mu^{2}=\lambda$ ) and assume that $x$ induces scalar multiplication by $\mu$ on each $V_{k}, k \geq 2$. Thus, $x$ (represented by $\operatorname{diag}(-\mu, \mu, \ldots, \mu))$ has determinant $-\mu^{2 m}$ and so (since $\left.x \in G^{\prime}\right), \mu^{2 m}=-1$. Then $\mu \neq \pm 1$ and so $V$ is not an orthogonal space.

Suppose that $V$ is symplectic or unitary. If $u, v \in V_{2}$ such that $(u, v) \neq 0$, then $(u, v)=\left(u^{x}, v^{x}\right)=(\mu u, \mu v)=\mu^{q+1}(u, v)$. Therefore, $\mu^{q+1}=1$ and so $\left(u_{1}, v_{1}\right)=\left(u_{1}^{x}, v_{1}^{x}\right)=\left(-\mu u_{1}, \mu v_{1}\right)=-\mu^{q+1}\left(u_{1}, v_{1}\right)=-\left(u_{1}, v_{1}\right)$. Hence, $\left\langle u_{1}\right\rangle \perp$ $\left\langle v_{1}\right\rangle$ and so $V=\left\langle u_{1}\right\rangle \perp\left\langle u_{1}\right\rangle^{\perp}$. In particular, since $V$ is non-degenerate, it can not be a symplectic space.

If $V$ is a unitary space, the fact that $\mu^{q+1}=1$ but $\mu^{2 m}=-1$ implies that $q \equiv-1 \bmod 4$. On the other hand, if $G$ is the general linear group (so $|F|=q$ ), the fact that $\left(\mu^{m}\right)^{2}=-1$ implies that $q \equiv 1 \bmod 4$. Let $G_{2}$ be the corresponding 2-dimensional classical group. If $D_{2}$ is the group of $2 \times 2$ diagonal matrices in $G_{2}$ whose diagonal entries are 2-elements of $F^{\times}$and $y_{0}=\left(\begin{array}{cc}0 & 1 \\ 1 & 0\end{array}\right)$, the semidirect product $Q=\left[D_{2}\right]\left\langle y_{0}\right\rangle$ is a Sylow 2-subgroup of $G_{2}$. Moreover, the matrices $\left(\begin{array}{cc}-\mu & 0 \\ 0 & \mu\end{array}\right)$ and $\left(\begin{array}{cc}0 & \mu \\ \mu & 0\end{array}\right)$ are conjugate in $G_{2}$ (via $\alpha\left(\begin{array}{cc}1 & 1 \\ 1 & -1\end{array}\right)$ where, in the unitary case, $\alpha^{q+1}=2^{-1}$ ) and so $x$ (represented by $\operatorname{diag}(-\mu, \mu, \ldots, \mu)$ ) is $G$-conjugate to a non-diagonal element of $S$. But since $S \cong Q \imath T_{m}$, if $D$ is the subgroup of diagonal matrices in $S$, then $x \in D \unlhd S$ and so all $S$-conjugates of $x$ are diagonal. But then $\bar{x}=x Z(G)$ cannot be unfused in $G / Z(G)$.

Therefore, we may assume that condition (b) of Lemma 4.5 holds for some pair $V_{i}, V_{j}$ which, without loss of generality, we may assume are $V_{1}$ and $V_{2}$. Thus, $V$ is an orthogonal space with all $V_{i}$ 's isometric to $\Pi^{\epsilon}, x$ is an involution and if $E_{1}$ and $E_{-1}$ are the eigenspaces of $x$ in $V$,

$$
\left(V_{1} \perp V_{2}\right) \cap E_{1} \cong\left(V_{1} \perp V_{2}\right) \cap E_{-1} \cong \Pi^{-\epsilon}
$$

and

$$
V_{1} \cap E_{1} \cong V_{1} \cap E_{-1} \neq V_{2} \cap E_{1} \cong V_{2} \cap E_{-1} .
$$

If $j \geq 3$, we have shown that conclusion (a) of Lemma 4.5 can not hold for either $V_{1} \perp V_{j}$ or $V_{2} \perp V_{j}$. But condition (b) can hold for at most one of these subspaces (because there are only two isometry classes of one-dimensional orthogonal spaces), and so it follows that $V_{j} \leq E_{ \pm 1}$. Moreover, if $j, k \geq 3$, neither (a) nor (b) holds for $V_{j} \perp V_{k}$ and so $x$ must induce the same scalar transformation on both summands. Replacing $x$ by $-x$ if necessary, we may assume that $x$ induces the identity map on the subspace $V_{3} \perp \ldots \perp V_{m}$.

It follows that if $[V, x]=\left\{-v+v^{x}: v \in V\right\}$, then $[V, x]=\left(V_{1} \perp V_{2}\right) \cap E_{-1}=$ $\left(V_{1} \cap E_{-1}\right) \perp\left(V_{2} \cap E_{-1}\right)$. Let $V_{1} \cap E_{-1}=\left\langle u_{1}\right\rangle$ and $V_{2} \cap E_{-1}=\left\langle u_{2}\right\rangle$. Since $\left\langle u_{1}\right\rangle$ and $\left\langle u_{2}\right\rangle$ are not isometric, we may assume that $\left(u_{1}, u_{1}\right) \in\left(F^{\times}\right)^{2}$ (and so after rescaling, $\left.\left(u_{1}, u_{1}\right)=1\right)$ and $\left(u_{2}, u_{2}\right)=c \in F \backslash\left(F^{\times}\right)^{2}$. If $\chi$ denotes the Wall form of $x\left([9]\right.$, p. 153), then since $u_{i}=\frac{1}{2}\left(u_{i}-u_{i}^{x}\right)$ for $i=1,2$, $\chi\left(u_{i}, v\right)=\frac{1}{2}\left(u_{i}, v\right)$ for all $v \in[V, x]$ and in particular, the matrix of $\chi$ with respect to $\left\{u_{1}, u_{2}\right\}$ is $X=\left(\begin{array}{cc}\frac{1}{2} & 0 \\ 0 & \frac{c}{2}\end{array}\right)$. The spinor norm of $x$ (the discriminant of $\chi)$ is $(\operatorname{det} X) F^{2}=\frac{c}{4} F^{2} \neq F^{2}$ and so $x \notin G^{\prime}$ ([9], Theorem 11.51). This contradiction completes the proof of the theorem.

Corollary 4.7 No non-abelian simple group of classical type in odd characteristic contains an unfused involution. 
Proof. Theorem 1 and Corollary 4.4.

\section{The exceptional groups in odd characteristic}

Assume that a minimal counterexample to the Main Theorem is an exceptional simple group of Lie type in odd characteristic. $G$ cannot be one of $G_{2}(q)$, ${ }^{2} G_{2}\left(3^{2 m+1}\right)$ or ${ }^{3} D_{4}(q)$ since each contains a unique conjugacy class of involutions. In each of the remaining cases, there is an involution $z \in Z(S)$ such that $z \in L \unlhd C_{G}(z), C_{G}(z) / L$ and $Z(L)$ are cyclic and $L / Z(L)$ is as indicated in the following table ([1], [7]):

\begin{tabular}{cc}
\hline$G$ & $L / Z(L)$ \\
\hline \hline$F_{4}(q)$ & $P \Omega_{9}(q)$ \\
$E_{6}(q)$ & $P \Omega_{10}^{+}(q)$ \\
${ }^{2} E_{6}(q)$ & $P \Omega_{10}^{-}(q)$ \\
$E_{7}(q)$ & $L_{2}(q) \times P \Omega_{12}^{+}(q)$ \\
$E_{8}(q)$ & $P \Omega_{16}^{+}(q)$
\end{tabular}

Except in the case $G=E_{7}(3)$, we conclude from Lemma 2.4 that $x \in Z(L)$, whence $x=z \in Z(S)$, a contradiction. If $G=E_{7}(3)$, then $L / O_{2,2^{\prime}}(L) \cong$ $P \Omega_{12}^{+}(3)$ and Corollary 2.5 implies that $x \in O_{2}(L)$. But $S L_{2}(3)$ has a unique involution and so we again have the contradiction $x=z \in Z(S)$.

\section{The groups of Lie type in characteristic 2}

We assume in this section that the minimal counterexample $G$ is a simple group of Lie type over the field $F_{q}$ of order $q=2^{k}$.

Let $x \in S \in S y l_{2}(G)$ and $B=N_{G}(S)$. Let $N \leq G$ such that $(G, B, N, \Sigma)$ is a Tits system (with $B$ and $N$ a $(B, N)$ )-pair and $\Sigma=\left\{w_{i}: i \in I\right\}$ a distinguished set of involutary generators of the Weyl group $W=N / B \cap N)$. I may, of course, be identified with a base of the corresponding root system.

Lemma 6.1 If $L$ is a centerless finite group of Lie type in characteristic 2 then either $L^{\prime}$ is simple or $L$ is solvable and isomorphic to one of $A_{1}(2), D_{2}(2)$, ${ }^{2} A_{2}(2)$ or ${ }^{2} B_{2}(2)$. In the solvable cases, $L^{\prime}$ has odd order unless $L \cong{ }^{2} A_{2}(2)$. (This exception is a Frobenius group of order 72 with a quaternion Sylow 2subgroup.)

Proof. See Proposition 2.9.2 and Theorem 5.1.1 of [8].

Lemma 6.2 Let $P$ be a maximal parabolic subgroup of $G$ containing $B$ and let $L$ be a Levi factor of $P$, so $O^{2^{\prime}}(L)$ is a central product of groups of Lie type in characteristic 2. If ${ }^{2} A_{2}(2)$ does not appear as a factor in this decomposition of $O^{2^{\prime}}(L)$, then $x \in O_{2}(P)$ and $P=N_{G}\left(\left\langle x^{S}\right\rangle\right)$.

Proof. If $\bar{P}=P / O_{2}(P)$, then $\bar{P} \cong L$, whence $O^{2^{\prime}}(\bar{P} / Z(\bar{P}))=O^{2^{\prime}}(\bar{P}) Z(\bar{P}) / Z(\bar{P})$ is a direct product $L_{1} \times L_{2} \times \ldots \times L_{k}$ of centerless groups of Lie type in characteristic 2. By the preceding lemma, $O^{2^{\prime}}(\bar{P} / Z(\bar{P}))^{\prime}=L_{1}^{\prime} \times L_{2}^{\prime} \times \ldots \times L_{k}^{\prime}$ where each $L_{i}^{\prime}$ is either simple or of odd order. Moreover, since $P \geq B \geq S, \bar{x} Z(\bar{P})$ is an unfused element of $O^{2^{\prime}}(\bar{P} / Z(\bar{P}))$ and so by Corollary 2.5, $x \in O_{2}(P)$ and $P \leq N_{G}\left(\left\langle x^{S}\right\rangle\right)$. By the maximality of $P, P=N_{G}\left(\left\langle x^{S}\right\rangle\right)$. 
Lemma 6.3 $N_{G}\left(\left\langle x^{S}\right\rangle\right)$ is the unique maximal parabolic of $G$ containing B.

Proof. Let $P$ be a maximal parabolic containing $B$. By the previous lemma, it is enough to show that no $L_{i}$ is isomorphic to ${ }^{2} A_{2}(2)$.

Suppose $L_{i} \cong{ }^{2} A_{2}(2)$ for some $i$. There is a lattice isomorphism between subsets $J$ of $I$ and parabolics $P_{J}$ of $G$ containing $B$ (e.g. [2], Theorem 8.3.4). Identifying $I$ with the nodes of the Dynkin diagram for $G$, for any $J \subseteq I$ the types of factors in the central product decomposition of $O^{2^{\prime}}\left(L_{J}\right)$ (where $L_{J}$ is a Levi factor of $P_{J}$ ) are those of the components of the subdiagram whose nodes correspond to elements of $J$. Therefore, $G$ must be a group of twisted type, consisting of elements of a Chevalley group $H$ (over a finite extension of $F_{q}$ ) fixed by a certain automorphism $\gamma$ which induces a symmetry of the Dynkin diagram of $H$. The parabolics of $G$ containing $B$ correspond to $\gamma$-invariant sets of nodes of this diagram. Moreover, a Levi factor of the parabolic corresponding to such a set $J$ consists of the elements fixed by $\gamma$ in a Levi factor of the parabolic of $H$ corresponding to $J$. (See [2], Chapter 13 and [8], p.180-181.)

Two nodes connected by a single bond lie in the same orbit of a symmetry of a Dynkin diagram only for diagrams of type $A_{2 m}$ and hence, we conclude that $H \cong A_{2 m}(2)$, and $G \cong{ }^{2} A_{2 m}(2), m \geq 2$. Moreover, of the $m$ maximal parabolics of ${ }^{2} A_{2 m}(2)$ containing a particular Borel subgroup $B,{ }^{2} A_{2}(2)$ appears in only one. (If the nodes of the Dynkin diagram $A_{2 m}$ are sequentially labelled $1,2, \ldots, 2 m$, this parabolic has a Levi factor of type $A_{1}\left(2^{2}\right) \times \ldots \times A_{1}\left(2^{2}\right) \times$ ${ }^{2} A_{2}(2)$ and corresponds to the omission of the nodes labelled $m-1$ and $m+2$.) By the previous lemma, $m-1=1$ and so $G \cong{ }^{2} A_{4}(2) \cong P S U_{5}(2)$.

A Sylow 2-subgroup of $\mathrm{PSU}_{5}(2)$ contains only one class $\mathrm{C}$ of non-central involutions and so we may assume that $x \in S \cap C$. Moreover, there is an elementary abelian subgroup $X \leq S$ of order $2^{4}$ which contains 10 involutions in $C$ (e.g. [4]), and so we may assume that one of these is $x$. Then for some $y \in X \cap C, x y$ is also in $X \cap C$ and so $x, y$ and $x y$ are all conjugate in $S$. This contradicts Lemma 2.1 and completes the proof of the lemma.

The contradiction. If $G$ is a Chevalley group (i.e. of untwisted type) then because of the lattice isomorphism between parabolics containing $B$ and subsets of $I$, the previous lemma implies that $|I|=1$ and so $G=A_{1}(q) \cong L_{2}(q), q=2^{k}$. But this group has abelian Sylow 2-subgroups and so, by Lemma 2.3, is not a counterexample to the Main Theorem.

If $G$ is a twisted group corresponding to a Chevalley group $H$ and an automorphism $\gamma$, then the previous lemma implies that the symmetry induced by $\gamma$ on the Dynkin diagram of $H$ is transitive on the nodes. Therefore, $H=A_{2}(q)$, $B_{2}(q)$ or $D_{2}(q), q=2^{k}$ and correspondingly, $G={ }^{2} A_{2}(q) \cong P S U_{3}(q),{ }^{2} B_{2}(q) \cong$ $S z(q)$ or ${ }^{2} D_{2}(q) \cong P \Omega_{4}^{-}(q) \cong L_{2}\left(q^{2}\right)$. The last of these groups has abelian Sylow 2-subgroups while those of the first two are of nilpotence class 2. Again, this contradicts Lemma 2.3 and so no counterexample to the Main Theorem is to be found among groups of Lie type in characteristic 2 .

\section{The sporadic groups}

Assume that the sporadic group $G$ is a minimal counterexample to the Main Theorem with unfused involution $x \in S \in S y l_{2}(G)$. The sole reference here will be the Atlas [4]. Immediately eliminated are the groups with a unique conjugacy class of involutions: $M_{11}, M_{22}, M_{23}, J_{1}, J_{3}, M c L, L y S, O^{\prime} N$ and $T h$. 
Note that by Lemma 2.1, $S$ cannot contain a fours subgroup all of whose involutions are conjugate in $S$ and so an unfused involution $x$ of $G$ cannot lie in a fours group $E \leq G$ all of whose involutions are $G$-conjugate. The groups $M_{12}, J_{2}, S u z, R u, H e, \mathrm{Co}_{3}, \mathrm{Fi}_{24}^{\prime}$ and $\mathrm{M}$ each have a unique class of involutions not in the center of a Sylow 2-subgroup and a fours group all of whose nonidentity elements are conjugate to a representative of this class so these groups are eliminated as possible counterexamples.

If $H$ and $K$ are maximal subgroups of odd index such that $\left(H / O_{2,2^{\prime}}(H)\right)^{\prime}$ and $\left(K / O_{2,2^{\prime}}(K)\right)^{\prime}$ are each direct products of non-abelian simple groups, Corollary 2.5 implies that $H=K$. Choosing $H$ and $K$ as indicated (in Atlas notation), this yields a contradiction for each sporadic group in the table below.

\begin{tabular}{ccccc}
\hline$G$ & $H$ & Structure of $H$ & $K$ & Structure of $K$ \\
\hline \hline$H S$ & $N\left(2 A^{3}\right)$ & $4^{3}: L_{3}(2)$ & $N(2 A)$ & $4.2^{4}: S_{5}$ \\
$M_{24}$ & $N\left(2 A^{4}\right)$ & $2^{4}: A_{8}$ & $N\left(2^{6}\right)$ & $2^{6}:\left(L_{3}(2) \times S_{3}\right)$ \\
$H N$ & $N(2 B)$ & $2_{+}^{1+8} \cdot\left(A_{5} \times A_{5}\right) .2$ & $N\left(2 B^{3}\right)$ & $2^{3} \cdot 2^{2} .2^{6} .\left(3 \times L_{3}(2)\right.$ \\
$C o_{2}$ & $N\left(2^{10}\right)$ & $2^{10}: M_{22}: 2$ & $N(2 A)$ & $2_{+}^{1+8}: P S p_{6}(2)$ \\
$F i_{22}$ & $N\left(2^{10}\right)$ & $2^{10}: M_{22}$ & $N\left(2 B^{4}\right)$ & $2^{5+8}:\left(S_{3} \times A_{6}\right)$ \\
$F i_{23}$ & $N(2 A)$ & $2 . F i_{22}$ & $N\left(2^{11}\right)$ & $2^{11} . M_{23}$ \\
$C o_{1}$ & $N\left(2^{11}\right)$ & $2^{11}: M_{24}$ & $N(2 A)$ & $2_{+}^{1+8}: P \Omega_{8}^{+}(2)$ \\
$J_{4}$ & $N\left(2^{11}\right)$ & $2^{11}: M_{24}$ & $N\left(2 A^{3}\right)$ & $2^{3+12} .\left(S_{5} \times L_{3}(2)\right)$ \\
$B$ & $N(2 B)$ & $2_{+}^{1+22} \cdot C o_{2}$ & $N\left(2 B^{8}\right)$ & $2^{9} .2^{16} . P S p_{8}(2)$
\end{tabular}

This exhausts the sporadic groups and eliminates the last of the possibilities for a counterexample to the Main Theorem.

\section{References}

[1] M. Aschbacher, A characterization of Chevalley groups over fields of odd order, Ann. Math. 106 (1977), 353-468.

[2] R. Carter, Simple Groups of Lie Type, Wiley-Interscience (1972)

[3] R. Carter and P. Fong, The Sylow 2-subgroups of the finite classical groups, J. Algebra 1 (1964), 139-151.

[4] J.H. Conway, R.T. Curtis, S.P. Norton, R.A. Parker and R.A. Wilson, An atlas of finite groups (Oxford University Press, 1985)

[5] S. Doro, Counterexamples on the fusion of involutions in finite groups, Proc. Amer. Math. Soc. 59 (1976), 23-24.

[6] G. Glauberman, Central elements in core-free groups, J. Algebra 4 (1966), 403-420.

[7] N. Iwahori, Centralizers of involutions in finite Chevalley groups, Seminar on Algebraic Groups and Related Finite Groups, Lecture Noters in Mathematics 131 (eds. A. Borel et al., Springer, Berlin (1970).

[8] P. Kleidman and M. Liebeck, The Subgroup Structure of the Finite Classical Groups, Cambridge University Press (1990).

[9] D. E. Taylor, The Geometry of the Classical Groups, Heldermann Verlag Berlin (1992). 